\title{
Wireless Biomedical Parameter Monitoring System Using Arm Microcontroller: A Review
}

\author{
Anju.C.K ${ }^{(1)}$, Lekshmi.S ${ }^{(2)}$ \\ ${ }^{(1)}$ Mtech student, VJEC chemperi,Kannur ${ }^{(2)}$ Assistant professor, VJEC Chemperi,kannur
}

\begin{abstract}
The main focus of the paper is to implement a model for the real time patient monitoring system. The proposed system is used to measure the physical parameters like body temperature, heartbeat, ECG, blood sugar, and oxygen level monitoring with the help of biosensors using arm microcontroller.There are number of techniques available for the ICU patient's health monitoring system with wired communication technology. In the novel system the patient health is continuously monitored and the acquired data is transmitted to an ARM server using wireless sensor networks.Here zigbee wireless sensor networks are using for the purpose. Embedded processor supports for analyzing the input from the patient and the results of all the parameters are stored in the database. If any abnormality felt by the patient automatic alarm sound will arrive and the message will send automatically the doctor mobile by using GSM module.
\end{abstract}

\section{Keywords: - ARM Processor, sensors, Zigbee, GSM, Biomedical monitoring system}

\section{INTRODUCTION}

The electronics technology has entered almost in all aspects of day-to-day life, and the medical field is not exception for that. The need for well-equipped hospitals and diagnostic centers is increasingday by day as the people are becoming more conscious about their health problems. Now lets try to find some reasons behind the increasing percentage of the patients. In today's world the life has become very fast. The throat cut competition for success has made people to work for more than 10 hours per day. Also the factors like increasing population, increasing pollution has affected day-to-day life. The no.of vehicles and undisciplined traffic has invited the no. of accidents every day. Also the stress on the mind and brain popularly known as 'Blues' are demanding the need for the well-equipped hospitals and diagnostic centers. The today's hospitals are big and covering large areas in a building. They may occupy no.of floors in one building. Different wards are situated at different places such as men's ward, women's ward, maternity ward, general ward, special rooms and more importantly ICU's. Doctors need to keep monitoring all the patients in these wards continuously, and this requires more number of skilled nurses and other concerned employees. Its not feasible for the doctors to go to each ward and monitor each patient frequently say after each half an hour. Keeping all these aspects in the mind we have developed "BIOMEDICAL DATA TRANSMISSION SYSTEM" which can be used efficiently to get rid of the problems mentioned in above paragraph.

In this system we are continuously monitoring the patient's different parameters such as body temperature and transmitting this data to the doctor's cabin continuously. Such sensors can be used for each patient and the related data can be transmitted to the doctor's cabin. At the doctor's cabin he can download data into master card by just pressing one key. We have provided the PC interface in our system to view the records of different patients situated at different places. Again by just pressing the just one key the doctor can upload this data to PC from the master card.

In this way it is beneficial for the large hospitals situated in the large cities to use this system. By employing this system they can reduce their manpower. Also the overhead of the doctors can be reduced up to large extent and their precious time can be utilized for the some good cause. Also since there are no human factors for the monitoring and recording purpose the errors can be eliminated and accuracy will be increased. And thus overall efficiency of the concern hospital will be increased.

Thus our system proves to be a useful one, for the every large-scale hospital to cure the patients quickly and creating a healthy society.

Recently wireless sensors and sensor networks plays a vital role in the research, technological community. But there are different from traditional wireless networks as well as computer networks, today the progress in science and technology offers miniature, speed, intelligence, sophistication, and new materials at lower cost, resulting in the development of various high-performance smart sensing system. Many new research is focused at improving quality of human life in terms of health by designing and fabricating sensors which are either in direct contact with the human body (invasive) or indirectly (noninvasive)[5],. In the current proposed system the patient health is continuously monitored by the patient monitoring system and the acquired data is 
transmitted to a centralized ARM server using Wireless Sensor Networks. A Zigbee node is connected to every patient monitor system that consumes very low power and is extremely small in size.

This paper builds an independent system that automatically logs vital parameters of patients for easy access. The data is accessible to doctors through mobile device for convenience. Data of all patients is stored in a common database. A system to monitor the overall health of welfare facility, which needs constant care, has been reported. The host computer stores the data, which can be used to analyze the patient's overall health condition.

When the patient is in an emergency situation, such as falling or in an inactive state for more that the allotted time, the host computer automatically alerts the situation to the care staff by an alarm sound and also the message has been send to doctor through GSM module. These facts show an increasing demand for longterm health monitoring which is affordable, continuous, and unobtrusive, which will result in considerable impact on annual medical costs and health management. Wearable systems for continuous health monitoring are a key technology in helping the transition to more practical and affordable healthcare. It not only allows the user to closely monitor changes in her or his aimed to provide emergency assistance to senior citizens, rehabilitation patients, and medically physiological parameters but also provides feedback to help maintain an optimal health status [8],. The fundamental problem with this system is that when medical emergencies happen to the user, they are often unconscious and unable to press an "emergency alert button." There is no product on the market which does not require manual activation of the alarm and monitors a user's vital signs smartly, though research is currently undergoing [3]. This is the novel design goal of the work presented in this paper. The reported device consists of a wrist strap and a finger ring (circuitry). This allows the sensors to be mounted around the wrist, finger and the ARM unit connected via ribbon cable.

\section{LITERATURE SURVEY}

Care of critically ill patient, requires spontaneous \& accurate decisions so that life-protecting \& lifesaving therapy can be properly applied. Statistics reveal that every minute a human is losing his/her life across the globe. More close in India, everyday many lives are affected by heart attacks and more importantly because the patients did not get timely and proper help .This paper is based on monitoring of remote patients, after he is discharged from hospital. I have designed and developed a reliable, energy efficient remote patient monitoring system. It is able to send parameters of patient in real time. It enables the doctors to monitor patient's parameters (temp, heartbeat, ECG) in real time. Here the parameters of patient are measured continuously (temp, heartbeat, ECG) and wirelessly transmitted using Zigbee. In the field of health monitoring the current most important user groups are those aged 40 and more. The group of $40+$ users shows more diversity in their health conditions than younger people. There are ring-type pulses monitoring sensor available in the market in which the measured data are displayed in the LCD and cannot be transmitted out of the ring. Thus, it is not possible to continuously monitor the vital parameters such as temperature, pressure and pulse from a distant location. In a hospital either the nurse or the doctor has to move physically from one person to another for health check, which may not be possible to monitor their conditions continuously.

Thus any critical situations cannot be found easily unless the nurse or doctor checks the person's health at that moment. This may be a strain for the doctors who have to take care of a lot number of people in the hospital.

In order to keep in track of critical health conditions, a real time health monitoring system of patient based on Zigbee, GSM, and SMS is designed and developed in this project. This finds vast application in the remote places where the people are out of reach from the experienced doctors; keeping this factor in mind best effort is done to implement some of the basic test of pathological data on the system[3][5].Karandeep Malhi et al [1] develop Zigbee smart noninvasive wearable physiological parameters monitoring device has been developed and reported in this paper. The system can be used to monitor physiological parameters, such as temperature and heart rate, of a human subject. The system consists of an electronic device which is worn on the wrist and finger, by an at risk person. Using several sensors to measure different vital signs, the person is wirelessly monitored within his own home. An impact sensor has been used to detect falls. The device detects if a person is medically distressed and sends an alarm to a receiver unit that is connected to a computer. This sets off an alarm, allowing help to be provided to the user. Rubina.a.shaikh, et al [2] Design a module to monitoring of remote patients, after he is discharged from hospital. I have designed and developed a reliable, energy efficient remote patient monitoring system. It is able to send parameters of patient in real time. It enables the doctors to monitor patient's parameters (temp, heartbeat, ECG) in real time. Here the parameters of patient are measured continuously (temp, heartbeat, ECG) and wirelessly transmitted using Zigbee. B. sirisha et al [3] describes a solution for enhancing the reliability, flexibility by improving the performance and power management of the real-time multi-patient monitoring system (MPMS).

In the current proposed system the patient health is continuously monitored by the MPMS and the acquired data is transmitted to a centralized ARM server using Wireless Sensor Networks. A Zigbee node is connected to every patient monitor system which will send the patient's vital information .Upon system boot up, 
the mobile patient monitor system will continuously monitor the patients vital parameters like Heart Beat, body temperature etc and will periodically send those parameters to a centralized server using Zigbee node configured as co-coordinator. If a particular patient's health parameter falls below the threshold value, a buzzer alert is triggered by the ARM server. Along with a buzzer an automated SMS is posted to the pre-configured Doctors mobile number using a standard GSM module interfaced to the ARM server. There are some shortcomings present in existing system. Currently there are number of health monitoring systems available for the ICU patients which can be used only when the patient is on bed. This system is wired everywhere. The patient is monitored in ICU and the data transferred to the PC is wired [3]. Such systems become difficult where the distance between System and PC is more. The available systems are huge in size. Regula of patient is not possible once he/she is discharged from hospitals. These systems cannot be used at individual level. The other problem with these systems is that it is not capable of transmitting data continuously also range limitations of different wireless technologies used in the systems. So to overcome these limitations of systems I have proposed a new system. This system is able to transmit the parameters of patient continuously and over long distance wirelessly [5]. Due to which we would be able attend the patient immediately. Therefore by developing a system that can constantly measure the important parameters of patient's body and which can alert the closed ones and the doctor on any time when the patient's condition gets bad, this can really provide quick service and be beneficial in saving a lot of lives.

\section{EXISTING SYSTEM}

There are some shortcomings present in existing system. The patient is monitored in ICU and the data transferred to the PC is wired. Such systems become difficult where the distance between System and PC is more. The available systems are huge in size. Regular monitoring of patient is not possible once he/she is discharged from hospitals. These systems cannot be used at individual level. The other problem with these systems is that it is not capable of transmitting data continuously also range limitations of different wireless technologies used in the systems. So to overcome these limitations of systems we proposed a new system. Our system is able to transmit the parameters of patient continuously and over long distance in wireless medium. ZigBee is „Wireless Networking Technology" and is an established set of specifications for wireless personal area networking (WPAN), i.e., digital radio connections between computers and related devices. This kind of network eliminates use of physical data buses like USB and Ethernet cables. We are using ZNet 2.5 (Series 2) modules. The XBee ZNet 2.5 modules from Digi are more advanced than the popular XBee Series 1modules, but they require a little additional work to set up. Due to which we would be able attend the patient immediately. Therefore by developing a system that can constantly measure the important parameters of patient's body and which can alert the closed ones and the doctor on any time when the patient's condition gets bad, this can really provide quick service and be beneficial in saving a lot of lives.

\section{PROPOSED SYSTEM DESCRIPTION}

The proposed system is designed for monitor the patient is in any place. The system would constantly monitor important physical parameters like temperature, heartbeat, ECG, blood sugar, and would compare it against a predetermined value set and if these values cross a particular limit it would automatically alert the alarm and doctor via a SMS. This system provides a continuous health monitoring service. The data processed are transmitted by Zigbee wireless. Finally the received data is sent to the PC. The graphical user interface programs on the PC are coded using keil C software, Using GSM modem message is transmitted to the doctor mobile number when the measured temperature exceeds the allowable value or if the pulse measured is abnormal. GSM is abbreviated as Global System for Mobile Communication10. GSM modem has a slot for inserting SIM (Subscriber Identity Module). GSM network contains Mobile Station, Base station subsystem and Network subsystem. Mobile station contains IMEI number and SIM has IMSI number. Base station subsystem contains Base Transceiver Station which has antennas for communication and Base Station Controller which controls multiple base stations. Network subsystem contains VLR (Visitor Location Register), HLR (Home Location Register), AuC (Authentication Center) and EIR (Equipment Identity Register). MSC (Mobile Switching Center) is the major part which is the gate way for communication between mobile station and PSTN. HLR stores the information about the subscriber and the current location of subscriber. VLR provides the services to the subscribers of HLR who are visitor users. AUC gives the security of the user and to identify the location of the subscriber. EIR is also for security purpose and to identify the mobile station. MAX232 is connected to GSM modem so that it is useful for serial data transmission. OSS (Operation Support System) is used to control the traffic of users.

\section{HARDWARE DESCRIPTION}

It includes various sensors like Temperature sensor, Heart beat sensor, ECG sensor, Blood sugar sensor, ARM processor, display, buzzer, and Zigbee connector circuit. Wireless sensors nodes with a single 
accessing from one AP (access point) to another have addressed the usefulness of these sensors in sending and retrieving data. The proposed circuit has the ability to determine the patient's temperature in real-time status inside the hospital. Heart beat sensor is designed to give digital output of heart beat when a finger is placed inside the clip. This digital output can be connected to Zigbee and transmitted to ARM directly to measure the Beats per Minute (BPM) rate. The ECG (Electrocardiogram) records the pathway of electrical impulses through the heart muscle, and can be recorded on resting and ambulatory subjects, or during exercise to provide information on the heart's response to physical exertion. Zigbee is 'Wireless Networking Technology' and is an established set of specifications for wireless personal area networking (WPAN), i.e., digital radio connections between computers and related devices. This kind of network eliminates use of physical data buses like USB and Ethernet cables. Zigbee indoors can usually reach $400 \mathrm{~m}$ range.

The ARM TDMI-S is a special ARM purpose 32-bit microprocessor, offers high performance and very low power consumption. ARM architecture is based on RISC principles, instruction set and related decode mechanism are simpler than CISC Pipeline techniques employed ARM Processor supports both 32-bit and 16bit instructions via the ARM and Thumb instruction sets. The 5 parameters to be monitored are sensed using respective sensor and data is feed to ARM processor.

Care of critically ill patient, requires spontaneous \& accurate decisions so that life-protecting \& lifesaving therapy can be properly applied. Statistics reveal that every minute a human is losing his/her life across the globe. More close in India, everyday many lives are affected by heart attacks and more importantly because the patients did not get timely and proper help. This paper is based on monitoring of remote patients, after he is discharged from hospital. It is able to send parameters of patient in real time. It enables the doctors to monitor patient's parameters (temp, heartbeat, ECG) in real time. Here the parameters of patient are measured continuously (temp, heartbeat, ECG) and wirelessly transmitted using Zigbee. There is a vast growth of VLSI technology and GSM communication in these days. This project deals about the implementation of GSM technology in Medical applications. This wireless communications would not only provide them with safe and accurate monitoring but also the freedom of movement. In this, heart beat and temperature of patient are measured by using sensors as analog data, later it is converted into digital data using ADC which is suitable for wireless transmission using paging messages through GSM modem. AT89S52 micro controller device is used for temporary storage of the data used for transmission

\section{CONCLUSIONS}

In this proposed model of monitoring physiological parameters such as temperature, heartbeat, ECG, blood sugar, are more powerful than currently available system. Currently available systems for monitoring physiological signals suffer from technical limitations. The proposed system is an enormous improvement over existing commercial methods, the present system can support up to twenty patients with real-time, low-power, low-cost, long-distance, and dual-mode monitoring, from the above designed project. The keil C software is used for implementing the process and results were discussed. In future we can expand this system by using RFID technology; through this technology we can monitor the multiple numbers of patients. It may be a future work to develop another patient monitoring application code in that direction.

\section{REFERENCES}

[1] Karandeep Malhi, Subhas Chandra Mukhopadhyay, Fellow, IEEE, Julia Schnepper, Mathias Haefke, and Hartmut Ewald "A Zigbee-Based Wearable Physiological Parameters Monitoring System" IEEE sensors journal, vol. 12, no. 3, march 2012 online at http://ieeexplore.ieee.org

[2] b. sirisha, t.sraddha, k. vijayanand "Real-time multi-patient monitoring system using arm and wireless sensor network" International Journal of Communication Network Security, ISSN: 2231 - 1882, Volume-2, Issue-2, 2013

[3] Reza S. Dilmaghani, Hossein Bobarshad, M Ghavami, Sabrieh Choobkar, and Charles Wolfe "Wireless Sensor Networks for Monitoring Physiological Signals of Multiple Patients" IEEE transactions on biomedical circuits and systems, vol. 5, no. 4, august 2011 online at http://ieeexplore.ieee.org.

[4] Yadav Satyendra Satyanarayan, Yadav Raghvendra Satyanarayan, Deep H. Desai "Intelligent Wireless Emergency Alert System for Patient Monitoring using AT89S52 Microcontroller" International Journal of Advanced Research in Electrical, Electronics and Instrumentation Engineering Vol. 2, Issue 4, April 2013 online at www.ijareeie.com

[5] shyr-kuen chen, tsair kao, chia-tai chan, chih-ning huang, chih-yen chiang, chin-yu lai, tse-hua tung, and pi-chung wang "A reliable transmission protocol for zigbee-based wireless patient monitoring" IEEE transactions on information technology in biomedicine, vol. 16, no. 1, January 2012 Online at http://ieeexplore.ieee.org.

[6] Sergio Gonz'alez-Valenzuela, Min Chen, Senior Member, IEEE, and Victor C. M. Leung, Fellow, IEEE Mobility "Support for Health Monitoring at Home Using Wearable Sensors" IEEE TRANSACTIONS 
ON INFORMATION TECHNOLOGY IN BIOMEDICINE, VOL. 15, NO. 4, JULY 2011 online at http://ieeexplore.ieee.org.

[7] X. Wang and H. Qian, "Design and implementation of anycast services in ad hoc networks connected to IPv6 networks," J. Netw., vol. 5, pp. 403-410, 2010.

[8] Y.Gu, A. Lo, and I. G. Niemegeers, "A survey of indoor positioning systems for wireless personal networks," IEEE Commun. Surv. Tutorials, vol. 11, no. 1, pp. 13-32, First Quarter 2009. online at http://ieeexplore.ieee.org

[9] Juney M George1, Venugopal G "Design of an embedded platform for patient monitoring applications using $\mu \mathrm{c}$ os-ii" International Journal of Advanced Research in Electrical, Electronics and Instrumentation Engineering Vol. 2, Special Issue 1, December 2013 online at www.ijareeie.com

[10] Nagender Kumar Suryadevara, Student Member, IEEE, and Subhas Chandra Mukhopadhyay, Fellow, IEEE "Wireless Sensor Network Based Home Monitoring System for Wellness Determination of Elderly" IEEE SENSORS JOURNAL, VOL. 12, NO. 6, JUNE 2012 